OPEN ACCESS

Edited by:

Feng-Huei Lin,

National Taiwan University, Taiwan

Reviewed by:

Feza Korkusuz,

Hacettepe University, Turkey

Guo-Chung Dong,

National Health Research Institutes,

Taiwan

${ }^{*}$ Correspondence:

Yong Jiao

cuidong8@foxmail.com

Bo Zhang

zhangbo@fmmu.edu.cn

Specialty section:

This article was submitted to

Tissue Engineering and Regenerative

Medicine,

a section of the journal Frontiers in Bioengineering and

Biotechnology

Received: 08 July 2021

Accepted: 29 November 2021

Published: 03 January 2022

Citation:

Cui $D$, Wu B, He D, Wang Y, Jiao Y and

Zhang B (2022) 3D-Printed Cold

Preservation Device in Renal Autotransplantation for the Treatment of a Patient With Renal Artery Stenosis. Front. Bioeng. Biotechnol. 9:738434.

doi: 10.3389/fbioe.2021.738434

\section{D-Printed Cold Preservation Device in Renal Autotransplantation for the Treatment of a Patient With Renal Artery Stenosis}

\author{
Dong Cui ${ }^{1}$, Bin $W_{u^{1}}$, Dali He ${ }^{1}$, Yanen $W^{2} g^{2}$, Yong Jiao ${ }^{1 *}$ and Bo Zhang ${ }^{1 *}$ \\ ${ }^{1}$ Department of Urology, Tangdu Hospital, The Air Force Medical University, Xi'an, China, ${ }^{2}$ Industry Engineering Department, \\ School of Mechanical Engineering, Northwestern Polytechnical University, Xi'an, China
}

Percutaneous transluminal angioplasty (PTRA) is a common treatment method for renal vascular disease (RVD). However, PTRA may not be effective in patients with abnormal vascular disease. Renal autotransplantation (RAT) has been used as an alternative therapy for these diseases. Restrictions due to intracorporeal kidney cold preservation and the renal function of intracorporeal RAT were not as well protected compared with open operation. We developed this technique of 3D-printed polylactide (PLA) cold jackets for laparoscopic complete intracorporeal RAT for the purpose of better protecting the renal function and determining the feasibility of this novel procedure. The procedure was successfully applied to a 51-year-old woman with bilateral renal artery stenosis. The operation time was 5 hours, and blood loss was $200 \mathrm{ml}$. The patient's blood pressure remained constant throughout the operation, and the pressure was maintained at 120140/70-90 mmHg without antihypertensive drugs 1 week after the operation. B-ultrasound showed that the blood flow signal of the transplanted kidney was normal and the boundary between the skin and medulla was clear. The patient was discharged 2 weeks after surgery. One year postoperatively, Doppler ultrasound of the autotransplant showed that the transplanted kidney was normal in size and shape. Radionuclide renal dynamic imaging revealed that the glomerular filtration rate (GFR) of the transplanted kidney was $36.9 \mathrm{ml} / \mathrm{min}$. 3D-printed polylactide (PLA) cold jackets for laparoscopic complete intracorporeal RAT are a safe and effective method for the treatment of renal artery stenosis and represent a feasible method for preserving the renal function of severe renal artery stenosis patients; however, the technology is still at the exploratory stage and has room for further improvements.

Keywords: 3D print, polylactide, kidney diseases, kidney transplantation, laparoscopic surgery, rat

\section{INTRODUCTION}

3D printing technology is a new manufacturing process that gradually developed after the $1980 \mathrm{~s}$. 3D printing (rapid prototyping or additive manufacturing) is the process of creating solid 3D objects from a collection of images in the form of a digital file. The printer deposits layers of materials (such as plastic, resin, or metal) in a volumetric manner such that a replica of the object is obtained. This innovative technology will likely revolutionize our knowledge and understanding of the structure of 
human tissues and organs, and it also has therapeutic applications. In recent years, as biomedical polymer materials have been widely used in the field of medicine, the use of $3 \mathrm{D}$ printing technology to prepare biomedical polymer materials has attracted the attention of many researchers and achieved important results, such as bone repair projects in orthopedics and neurology, nerve tissue engineering scaffold material projects, and surgical planning projects. These projects have produced ideal repair effects and greatly reduced medical costs.

Renal vascular disease (RVD) is a rare but important cause of hypertension. Because essential hypertension is increasingly common and blood pressure is not commonly measured, RVD is not usually diagnosed at an early stage. Percutaneous transluminal angioplasty (PTRA) is a common treatment method for RVD (Buck et al., 2016), and this technology has improved over time and is now increasingly used, such as for placing vascular stents at the level of artery stenosis after forced dilation in a balloon (Kari et al., 2015; Chavent et al., 2017; Agrawal et al., 2018; Bacic et al., 2020; Raborn et al., 2020). This method may provide a minimally invasive alternative for certain patients who appear to be resistant to PTRA (Kim et al., 2017; Safdar et al., 2020).

However, PTRA may not be effective and could even be dangerous in patients with dysplasia or atherosclerotic lesions involving infrahilar branches or inflammatory lesions, such as those associated with Takayasu disease (TD). Therefore, the role of PTRA in the treatment of renovascular hypertension $(\mathrm{RVH})$ is still controversial, especially in patients who present complicated renal vascular lesions or patients for whom antihypertensive drugs either are ineffective or carry a potential risk of acute renal failure.

At present, renal autotransplantation (RAT) has been used as an alternative therapy for renovascular hypertension. Traditional renal autotransplantation using an open technique with one or two incisions is required for nephrectomy or anastomosis of renal vessels. Currently, the benefits of laparoscopic surgery are well established and have led to its widespread adoption, including in the field of transplantation (Giacomoni et al., 2016; Cantrell and Oberholzer, 2018; Choi et al., 2018; Perkins et al., 2018; Spinoit et al., 2019). Some studies reported the use of laparoscopic and robot renal autotransplantation (RAT). In most studies, the use of laparoscopy is limited and mostly applied for nephrectomy (Hiess and Seitz, 2016; Siena et al., 2019; Kishore et al., 2020). After nephrectomy, renal vessels are extracted from the abdominal cavity and transferred to the bench table, and a separate incision is required to anastomose the renal vessels with the iliac vessels (Kubota et al., 2020; Pomy et al., 2020; Smith et al., 2020). Although some studies have reported intracorporeal robotassisted renal autotransplantation and restrictions due to kidney cold preservation in vivo, the kidney is not cold preserved continuously during the operation after vessel dissociation (Breda et al., 2021). The results of these studies suggest that compared with intracorporeal renal autotransplantation, faster renal function recovery and shorter cold ischemia time occur with extracorporeal robot-assisted renal autotransplantation, suggesting that in intracorporeal renal autotransplantation, renal function was not best protected. At present, the use of continuous cold preservation in laparoscopic complete intracorporeal of human renal autotransplantation has not been reported.

Hypothermic perfusion and preservation technology are necessary conditions for renal autotransplantation. Cold perfusion can provide sufficient time and an appropriate operating environment for autologous kidney transplantation. Hypothermic perfusion fluid can protect renal function during renal ischemia (Lin et al., 2020), but one disadvantage of this method of continuous perfusion is that the effect is not accurate, and cell necrosis, apoptosis, and acute tubular necrosis are caused by blood supply recovery, especially when blood vessel anastomosis cannot be carried out (Urbanellis et al., 2020). The common method is adding ice water during suture, but continuous insertion of ice slush in the abdominal cavity not only distracts the surgeon and increases operative time but also is a rudimental method that does not guarantee a homogeneous parenchymal cooling effect with the possibility of compromising graft function. Cold ischemia preservation is the best preservation method (Kaths et al., 2017). A device covering the whole renal surface isolates the kidneys from surrounding tissue and maintains a constant low temperature, potentially preserving the renal function for a longer time, and by an insulation layer, it avoids cooling-related complications to the surrounding abdominal organs; this may be a perfect way to preserve the kidney. 3D solid models according to the shape of the kidney, and the greatest advantage of $3 \mathrm{D}$ printing is that artificial implants can be customized accurately and effectively. So we use 3D printing technology to develop a cooling jacket that consists of two sealed films that completely cover the kidney to form a channel for the cooling solution from one end to the other, and apply cold ischemia preservation continuously for the kidney.

This 3D-printed cold jacket can completely cover the graft, avoid the gradual melting of ice in the abdominal cavity, control the kidney temperature, and reduce the potential graft impairment, and it can be continued during the process of suturing. Its effect may be equivalent to that of cold preservation in vitro. Currently, some studies reported the use of cold preservation devices for pig intraperitoneal kidney transplantation (Menon et al., 2014; Meier et al., 2018; Samuels et al., 2019). However, they are all in the exploratory stage. There were no reports on the use of this $3 \mathrm{D}$ printing device in completely minimally invasive human kidney transplantation.

Polylactide (PLA), a kind of linear thermoplastic aliphatic polyester, is mainly prepared from starch raw materials through saccharification, fermentation, and certain chemical reactions. PLA has good biocompatibility and biodegradability and can be completely degraded under specific conditions, and the final products are carbon dioxide and water (Madhavan Nampoothiri et al., 2010; Tyler et al., 2016). In addition, PLA also has good thermal stability, solvent resistance, excellent gloss, transparency, resistance to certain bacteria, and flame retardancy (Farah et al., 2016). Based on the advantage of its unique plasticity and transparency, 3D-printed polylactide (PLA) cold jackets can be used completely intracorporeally. We can clearly observe the kidney condition and the color of the kidney during the whole process of vascular anastomosis, and it is convenient for 


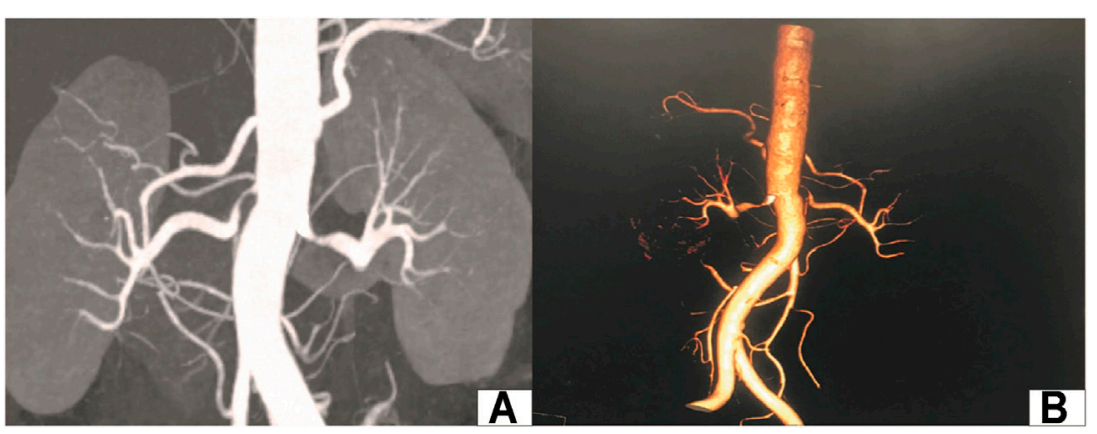

FIGURE 1 | Preoperative images of the renal artery. (A): CT renal angiography; (B): CT 3D reconstruction of renal vessels.

operation and can apply cold ischemia preservation continuously during the operation after vessel dissociation; thus, the kidney function can be protected to the maximum extent. This technology may make complete intracorporeal renal autotransplantation convenient, effective, and safe. Such surgery may be unique with no incision to extract or introduce kidneys, and cold ischemia preservation can be applied as same as the in vitro approach. Currently, there have been no reports on the use of this continuous cold ischemia preservation technology in laparoscopic complete intracorporeal of human renal autotransplantation. We developed this 3Dprinted polylactide (PLA) cold jacket technique for completely intracorporeal laparoscopic RAT for the purpose to investigate the feasibility of this novel procedure and describe the first successful application of this concept in the treatment of renal artery stenosis.

\section{MATERIALS AND METHODS}

\section{Patient}

The patient is a 51-year-old woman who was admitted to the urology department of our hospital on February 21, 2017. The blood pressure of the patient was elevated and fluctuated in the range of 160-200/90-100 mmHg. Nifedipine and indapamide were used, which provided poor blood pressure control. Thyroid functions and blood biochemical examination showed no extensive abnormalities. The plasma renin activity, angiotensin, and aldosterone were normal. Ultrasonic examination showed that the inner diameter at the beginning of the left and right renal arteries was 2.2 and $1.5 \mathrm{~mm}$, respectively. Renal artery CTA showed $80 \%$ stenosis in the proximal segment of the left renal artery and 90\% stenosis in the initial segment of the right renal artery (Figures 1A,B). The CT examination of the adrenal glands showed no significant abnormalities in either adrenal glands. Bilateral radionuclide renal dynamic imaging showed that the glomerular filtration rate (GFR) of the left and right kidneys was $56.0 \mathrm{ml} / \mathrm{min}$ and $21.5 \mathrm{ml} / \mathrm{min}$, respectively. Complete intracorporeal laparoscopic right kidney renal autotransplantation was proposed. After the operation, the operation time, bleeding loss, hot ischemia time (from ligation of the right renal artery to initiation of renal cold



FIGURE 2 | 3D printing cold jacket of the kidney.

perfusion), cold ischemia time (from initiation of renal cold perfusion and preservation to completion of venous anastomosis), time of venous and arterial anastomosis, postoperative blood pressures, and GFR were recorded.

\section{D Printing}

Thin-layer CT scan images of the patients' kidneys were extracted, DICOM format files were extracted from the CT scans, and images were processed with Mimics 17.0 software (Materialise Inc., Leuven, Belgium). After using threshold selection, region growth, multilayer editing, and modification techniques, three-dimensional reconstruction images of different parts of the kidney were obtained and then combined into a complete kidney. Using Mimics 17.0 software (Materialise Inc., Leuven, Belgium) cold jackets were designed.

The cool jacket is $2 \mathrm{~mm}$ larger than the real kidney, which is convenient for operation. The cooling jacket consists of two sealed films that completely cover the kidney to form a channel for the cooling solution from one end to the other. The equipment connects inflow and outflow to form a circuit, and the cooling solution operates at a constant volume and temperature, isolating the kidneys from surrounding tissue, which avoids the injury of adjacent abdominal organs and the heat and excessive cold injury of the kidney. The transparent and elastic PLA material is used for 3D rinting, suitably maintains the 


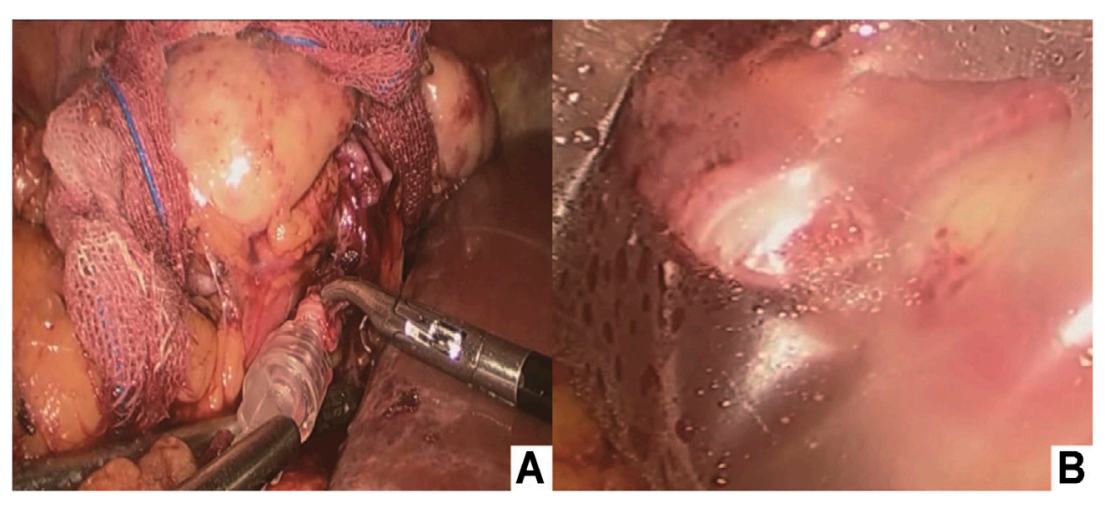

FIGURE 3 | (A) Intracorporeal cold perfusion of the kidney using a cannula and tubing passed through the assistant port to allow renal artery perfusion until clear effluent was seen from the renal vein and the color of the right kidney was pale; (B) 3D-printed cold jacket was completely covered on the surface of the free kidney.

temperature of the kidney, and allows easy extraction of the device. A window was designed to access the renal hilum for vascular anastomosis, while the kidney is still in the cold jacket. Finally, a standardized STL file of the cool jacket for 3D printing was output.

The STL files were formatted to meet printing parameters of the 3D printer (Shanghai liantai Technology Co., Ltd. rs4500 China). A PLA filament (Nature Work Inc., USA) is extruded from a $0.3-\mathrm{mm}$ nozzle at the optimum temperature of $210^{\circ} \mathrm{C}$. The external thickness of the cool jacket was $1.5 \mathrm{~mm}$, the internal thickness was $1 \mathrm{~mm}$, the distance between the two layers is $2 \mathrm{~mm}$, and the diameter of inflow and outflow pipes is $3 \mathrm{~mm}$. The printing time is 8 hours; after the printing, the cold jacket is rinsed in $70 \%$ ethanol overnight and sterilized for 90 min using the low-temperature plasma hydrogen peroxide sterilizer (Shandong Xinhua Medical Instrument Co., LTD., SQ-D, China) (Figure 2).

\section{Surgical Technique}

Complete intracorporeal laparoscopic right renal autotransplantation was performed. General anesthesia and indwelling catheter: a $10-\mathrm{mm}$ trocar was inserted at the outer edge of the rectus abdominis parallel to the umbilicus as the laparoscopic lens hole. Approximately $6 \mathrm{~cm}$ from the laparoscopic lens hole, two $10-\mathrm{mm}$ trocars were inserted at the hypogastrium and medial side of the anterior superior iliac spine (as an isosceles triangle). The right external iliac artery and vein, proximal to the bifurcation of the iliac vessels, distal to the abdominal wall, were carefully dissociated. Approximately $4 \mathrm{~cm}$ from the umbilicus at the edge of the rectus abdominis, a $10-\mathrm{mm}$ trocar was inserted as the laparoscopic lens hole, and approximately $3 \mathrm{~cm}$ from the costal margin, a $10-\mathrm{mm}$ trocar was inserted in the midclavicular line.

After the colon was dissociated, the renal vessels were completely dissociated to the maximum extent and completely dissociated from the right kidney. The excess perirenal fat was removed, and the fat that maintained the blood supply of the ureter between the lower pole of the kidney and the ureter was retained, and the adipose tissue around the ureter was properly retained. The ureter was distally dissociated as far as possible, and the upper and lower poles of the right kidney were bound with yarn strips as the extraction points of the kidney. From the root of the renal arteriovenous artery, the renal artery was ligated using laparoscopic clips close to the aorta, and the renal vein was then ligated in a similar manner. The renal artery and vein were then transected above the clips, and arteriovenous dissociation was carefully performed.

Immediately after dividing the vessels, the perfusion cannula was inserted in the transected artery lumen, which was continuously flushed with ice-cold perfusion liquid (hypertonic purine citrate fluid) solution under gravity until the clear effluent was seen from the renal vein and the color of the right kidney was pale (Figure 3A). The 3D-printed cold jacket completely covered the surface of the free kidney, and ice-cold saline water was continuously infused through the infusion pipeline. The kidney was carefully placed in the pelvis, and the opening of the renal artery and vein were placed close to the external iliac vessels (Figure 3B; Figure 4).

The external iliac vein was clamped with laparoscopic bulldog clamps, and a venotomy incision was made. A running end-to-side anastomosis was created between the renal vein and the external iliac vein using a 5-0 Prolene line. Before the last suture was placed, the lumen was irrigated with heparinized saline through a $5-\mathrm{Fr}$ ureteral catheter to remove intraluminal air. After completing the venous anastomosis, a bulldog clamp was placed on the renal vein, and the clamps were released from the external iliac vein. End-toside arterial anastomosis was performed, similar to that for the vein. The $3 \mathrm{D}$-printed cold jacket was then removed from the surface of the kidney. Upon completion, the clamps were removed, beginning with the distal external iliac artery, followed by the renal vein and then the proximal external iliac artery.

\section{RESULTS}

The operation time was 5 hours, and the hot ischemia time (from ligation of the right renal artery to initiation of renal cold perfusion) was $2 \mathrm{~min}$. The cold ischemia time (from initiation of renal cold perfusion and preservation to the completion of venous anastomosis) was $76 \mathrm{~min}$. The time for venous and arterial anastomosis was 23 and $27 \mathrm{~min}$, respectively. In addition, the estimated blood loss was $200 \mathrm{ml}$ (Table 1). The blood pressure 


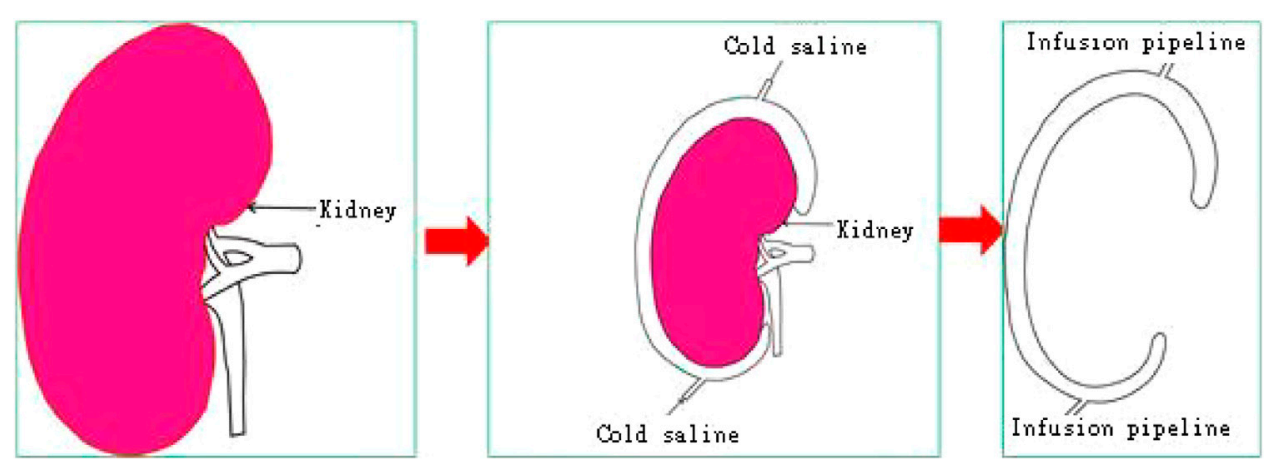

FIGURE 4 | 3D-printed model of the cold jacket covered on the surface of free kidney.

TABLE 1 | Outcomes of operation.

\section{Item}

Operation time

Blood loss

Hot ischemia time

Cold ischemia time

Venous anastomosis

Arterial anastomosis
Outcomes

$301 \mathrm{~min}$

$200 \mathrm{ml}$

$2 \mathrm{~min}$

$76 \mathrm{~min}$

$23 \mathrm{~min}$

$27 \mathrm{~min}$

remained constant throughout the operation. Postoperative rehydration and infection prevention were administered. Blood pressure monitoring was maintained at $120-140 / 70-90 \mathrm{mmHg}$ without antihypertensive drugs. The patient was discharged 2 weeks after surgery. One 1 year postoperatively, Doppler ultrasound of the autotransplant showed that the transplanted kidney was normal in size and shape, and the boundary between the skin and medulla was clear (Figure 5A). The blood flow signal of the transplanted kidney was not abnormal, the transplanted kidney was supplied by the right external iliac artery, and the vein returned to the right external iliac vein (Figure 5B). The arteriovenous phase and excretory phase were well developed. Radionuclide renal dynamic imaging revealed that the GFR of the transplanted kidney was $36.9 \mathrm{ml} / \mathrm{min}$.

\section{DISCUSSION}

Currently, renal autotransplantation (RAT) has been reported as an effective treatment model for managing complex renal/ureteral lesions. The first RAT in humans was performed by Hardy in 1963 to address an extensive ureteral lesion. Over the past 30 years, RAT has been used in the surgical management of complex ureteral lesions, renal artery aneurysms, $\mathrm{RVH}$, renal tumors, low back pain syndrome, and hematuria (Gwon et al., 2017; Ruiz et al., 2017; Doumerc et al., 2018; Haberal et al., 2018; Chen et al., 2020). RVH caused by renal artery stenosis presents as refractory hypertension and with poor drug treatment effect. It generally consists of percutaneous transluminal renal angioplasty (PTRA), in situ vascular reconstruction, or renal autotransplantation (RAT).

These procedures aim to normalize the blood flow to the affected kidney with the fewest possible surgical complications. However, PTRA may be unsuccessful and even hazardous in patients with dysplastic or atherosclerotic lesions involving infrahilar branches or with inflammatory lesions, such as those associated with TD. RAT has been used as an alternative treatment for the aneurysmal or complex occlusive disease of the renal artery. In the studied case of bilateral renal artery severe stenosis, stenosis of the right renal artery was more severe than that on the left, with the right renal artery accounting for approximately $90 \%$ of the stenosis. Narrowing was observed in the proximal renal artery, and because of the poor right kidney function, interventional therapy was difficult. Therefore, we selected RAT as an alternative treatment for this patient to obtain the best long-term results, improve the right kidney blood supply, and obtain compensatory right-side functions. If poor postoperative blood pressure control is achieved, then further treatment of left renal artery stenosis can be administered.

Laparoscopy and robot surgery have been used for donor nephrectomy and renal autotransplantation. Restrictions due to kidney cold preservation in vivo, most studies take the kidney extracted from the abdominal cavity and transfer to the bench table, and a separate incision is required to anastomose the renal vessels with the iliac vessels; the kidney is not cold preserved continuously during the operation (Ju et al., 2016; Kumar et al., 2018; Tiong et al., 2018; Ölçücüoğlu, 2020).Currently, some studies reported the use of cold preservation devices for pig intraperitoneal kidney transplantation (Menon et al., 2014; Meier et al., 2018; Samuels et al., 2019). However, they are all in the exploratory stage. In addition, some researchers reported using cold preserve in living donor's kidney transplants (Territo et al., 2021), but this method requires an additional incision to insert the cold preserve device and donor's kidney. There were no reports on the use of this device to human completely minimally invasive renal autotransplantation.

In this study, the patient underwent laparoscopic complete intracorporeal renal autotransplantation, the blood pressure was maintained at a normal level, the blood supply of the transplanted kidney recovered to normal levels, and the GFR was significantly recovered. Therefore, we believe that laparoscopic complete intracorporeal renal autotransplantation may be the preferred 




FIGURE 5 | Postoperative imaging confirmed autotransplantation success. (A) Doppler ultrasound of autotransplant showing that the transplanted kidney was of normal size and shape; $(\mathbf{B})$ the transplanted kidney was supplied by the right external iliac artery, and the vein returned to the right external iliac vein.

treatment for severe renal artery stenosis for which interventional therapy is not appropriate.

3D printing technology converts two-dimensional images into specified materials for printing through computer design software (Atalay et al., 2017; Ghazi et al., 2017; Bai et al., 2020). With the concept of biological manufacturing, the application of 3D printing technology in medicine has received increasing attention from researchers worldwide. At present, great progress has been made in reflecting the anatomical details of human organs by $3 \mathrm{D}$ printing, which was first used in preoperative planning and surgical simulation of complex operations (Ali et al., 2020; Xu et al., 2020). It has been more commonly used in orthopedics, stomatology, and cranial maxillofacial surgery. Giovinco (Giovinco et al., 2012) applied 3D printing technology in preoperative training for Charcot's foot orthopedic surgery and achieved good results. In the operation to repair an acetabular fracture, a simulated pelvis model of the patient was printed with $3 \mathrm{D}$ technology, and plate pre-bending, screw length measurement, and screw entry direction design were carried out on the model, which greatly reduced the operation time and surgical complications; moreover, the fracture model can be used to train new doctors. 3D printing technology has been applied in urology to print individual 3D solid models according to the shape of the kidney, and the greatest advantage of $3 \mathrm{D}$ printing is that artificial implants can be customized accurately and effectively. In this study, we used 3D printing technology to produce a temporary "cold jacket" for the kidney, and its effect was the same as that of in vitro cold preservation. This new method of $3 \mathrm{D}$ printing technology combined with cold preservation was first successfully applied in the treatment of renal artery stenosis, and this technology has not been previously reported.

PLA, a kind of linear thermoplastic aliphatic polyester, is mainly prepared from starch raw materials through saccharification, fermentation, and certain chemical reactions (Madhavan Nampoothiri et al., 2010; Tyler et al., 2016). It has good thermal stability, solvent resistance, excellent gloss, transparency, resistance to certain bacteria, and flame retardancy (Farah et al., 2016). Because of its unique performance, PLA is widely used in medical tissue engineering research, and it is feasible to make a cold storage device with this material. In this study, our novel cold storage devices overcome the restriction of kidney preservation in the process of complete intracorporeal renal autotransplantation. The kidney was preserved continuously, and the kidney function was protected to the maximum extent; this technology is safe and feasible for use in the process of human autologous kidney transplantation.

However, there are some limitations. First, this study applied $3 \mathrm{D}$ printing cold jackets for renal autotransplantation, and the proposed method was only applied to one patient. Additionally, although this new method is very promising, random comparisons with traditional renal autotransplantation were not performed, and the long-term effects and large sample outcomes have not been determined. Finally, whether the technique is suitable for different types of renal transplantation patients requires further clarification; thus, this cold jacket should be optimized in future research.

\section{CONCLUSION}

A 3D-printed polylactide (PLA) cold jacket for laparoscopic completely intracorporeal renal autotransplantation is a safe, effective, and less complicated method for the treatment of renal artery stenosis; however, the technology is at the exploratory stage and, thus, still has room for improvement. Every step still needs further research and elaboration, the cases need to be carefully selected, and the surgeon must be skilled in laparoscopic techniques, especially laparoscopy vascular sutures.

\section{DATA AVAILABILITY STATEMENT}

The original contributions presented in the study are included in the article/Supplementary Material, further inquiries can be directed to the corresponding authors.

\section{ETHICS STATEMENT}

The studies involving human participants were reviewed and approved by the Local Ethics Committee of Tangdu Hospital. The 
patients/participants provided their written informed consent to participate in this study. Written informed consent was obtained from the individual(s) for the publication of any potentially identifiable images or data included in this article.

\section{AUTHOR CONTRIBUTIONS}

DC and BZ contributed to the conception and design of the study, discussion of the results, and drafting of the manuscript. $\mathrm{HDL}, \mathrm{BW}$, and BZ were responsible for the diagnosis and treatment of the disease. YEW, YJ, and DC organized the follow-up research. YEW, YJ, and YCZ performed the imaging

\section{REFERENCES}

Ali, S., Sirota, E., Ali, H., Bezrukov, E., Okhunov, Z., Bukatov, M., et al. (2020). Three-Dimensionally Printed Non-Biological Simulator for Percutaneous Nephrolithotomy Training. Scand J. Urol. 54, 349-354. doi:10.1080/ 21681805.2020.1773529

Agrawal, H., Moodie, D., Qureshi, A. M., Acosta, A. A., Hernandez, J. A., Braun, M. C., et al. (2018). Interventions in Children with Renovascular Hypertension: A 27-year Retrospective Single-center Experience. Congenit. Heart Dis. 13, 349-356. doi:10.1111/chd.12608

Atalay, H. A., Canat, H. L., Ülker, V., Alkan, İ., Özkuvanci, Ü., and Altunrende, F. (2017). Impact of Personalized Three-Dimensional (3D) Printed Pelvicalyceal System Models on Patient Information in Percutaneous Nephrolithotripsy Surgery: a Pilot Study. Int. Braz. J. Urol. 43, 470-475. doi:10.1590/s16775538.ibju.2016.0441

Bacic, J., Liu, T., Thompson, R. H., Boorjian, S. A., Leibovich, B. C., Golijanin, D., et al. (2020). Emulating Target Clinical Trials of Radical Nephrectomy with or without Lymph Node Dissection for Renal Cell Carcinoma. Urology 140, 98-106. doi:10.1016/j.urology.2020.01.039

Bai, F., Wu, H., Zhang, N., Chen, J., and Wen, J. (2020). The Feasibility, Safety, and Efficacy of the Preemptive Indwelling of Double-J Stents in Percutaneous Nephrolithotomy Surgery: A Randomized Controlled Trial. Urol. J. 17, 232-236. doi:10.22037/uj.v0i0.4957

Breda, A., Diana, P., Territo, A., Gallioli, A., Piana, A., Gaya, J. M., et al. (2021). Intracorporeal versus Extracorporeal Robot-Assisted Kidney Autotransplantation: Experience of the ERUS RAKT Working Group. Eur. Urol. 12, S0302-S2838. doi:10.1016/j.eururo.2021.07.023

Buck, D. B., Curran, T., McCallum, J. C., Darling, J., Mamtani, R., van Herwaarden, J. A., et al. (2016). Management and Outcomes of Isolated Renal Artery Aneurysms in the Endovascular Era. J. Vasc. Surg. 63, 77-81. doi:10.1016/ j.jvs.2015.07.094

Cantrell, L. A., and Oberholzer, J. (2018). Robotic Pancreas Transplantation: the State of the Art. Curr. Opin. Organ. Transpl. 23, 423-427. doi:10.1097/ MOT.0000000000000555

Chavent, B., Duprey, A., Lavocat, M.-P., Fichtner, C., Beraud, A.-M., Albertini, J.N., et al. (2017). Renovascular Hypertension: Results in Adulthood of Renal Autotransplantation Performed in Children. Pediatr. Nephrol. 32, 1935-1940. doi:10.1007/s00467-017-3664-X

Chen, X., Zhao, J., Yuan, D., Yang, Y., and Huang, B. (2020). Restenosis of Bilateral Aorta-Renal Saphenous Vein Grafts after the Surgical Repair of Takayasu Arteritis-Induced Bilateral Renal Arteries Stenosis: Case Report. Ann. Vasc. Surg. 62, e1-498. doi:10.1016/j.avsg.2019.06.021

Choi, C. I., Kim, D. I., Baek, S. H., Chung, Y. S., Kim, D. H., Jeon, T. Y., et al. (2018). Initial Experience with Hand-Assisted Laparoscopic Living Donor Nephrectomy: Training and Clinical Practice as a General Surgeon. Transplant. Proc. 50, 3113-3120. doi:10.1016/j.transproceed.2018.08.052

Doumerc, N., Beauval, J.-B., Roumiguié, M., Roulette, P., Laclergerie, F., Sallusto, F., et al. (2018). Total Intracorporeal Robotic Renal Auto-Transplantation: A New Minimally Invasive Approach to Preserve the Kidney after Major Ureteral Injuries. Int. J. Surg. Case Rep. 49, 176-179. doi:10.1016/j.ijscr.2018.06.017 examinations and data collection. DC, BZ, and YEW carried out the 3D modeling, data post-processing, and stent manufacturing. All authors contributed to the article and approved the submitted version.

\section{ACKNOWLEDGMENTS}

We are grateful for the teams of the Department of Radiology and Department of Ultrasound Diagnostics (Tangdu Hospital) for identifying potential research participants and providing facilities. We would also like to thank Prof. He Wang for providing helpful advice and suggestions.

Farah, S., Anderson, D. G., and Langer, R. (2016). Physical and Mechanical Properties of PLA, and Their Functions in Widespread Applications - A Comprehensive Review. Adv. Drug Deliv. Rev. 107, 367-392. doi:10.1016/ j.addr.2016.06.012

Ghazi, A., Campbell, T., Melnyk, R., Feng, C., Andrusco, A., Stone, J., et al. (2017). Validation of a Full-Immersion Simulation Platform for Percutaneous Nephrolithotomy Using Three-Dimensional Printing Technology. J. Endourol. 31, 1314-1320. doi:10.1089/end.2017.0366

Giacomoni, A., Di Sandro, S., Lauterio, A., Concone, G., Buscemi, V., Rossetti, O., et al. (2016). Robotic Nephrectomy for Living Donation: Surgical Technique and Literature Systematic Review. Am. J. Surg. 211, 1135-1142. doi:10.1016/ j.amjsurg.2015.08.019

Giovinco, N. A., Dunn, S. P., Dowling, L., Smith, C., Trowell, L., Ruch, J. A., et al. (2012). A Novel Combination of Printed 3-dimensional Anatomic Templates and Computer-Assisted Surgical Simulation for Virtual Preoperative Planning in Charcot Foot Reconstruction. J. Foot Ankle Surg. 51, 387-393. doi:10.1053/ j.jfas.2012.01.014

Gwon, J. G., Kim, Y. H., and Han, D. J. (2017). Real Renal Function after Renal Autotransplantation through the Analysis of Solitary Kidney Autotransplantation Cases. Transplant. Proc. 49, 2055-2059. doi:10.1016/ j.transproceed.2017.09.030

Haberal, H. B., Tonyali, S., Peynircioğlu, B., Arici, M., Demircin, M., and Aki, F. T. (2018). Renal Autotransplantation with Autologous Saphenous Vein Graft in a Patient with Takayasu Arteritis and Existing Renal Artery Stent in Her Solitary Kidney. Urol. Int. 100, 181-184. doi:10.1159/000475509

Ju, X., Li, P., Shao, P., Lv, Q., Wang, Z., Qin, C., et al. (2016). Retroperitoneal Laparoscopic Nephrectomy Combined with Bench Surgery and Autotransplantation for Renal Cell Carcinoma in the Solitary Kidney or Tumor Involving Bilateral Kidneys: Experience at a Single Center and Technical Considerations. Urol. Int. 97, 473-479. doi:10.1159/000448594

Kari, J. A., Roebuck, D. J., McLaren, C. A., Davis, M., Dillon, M. J., Hamilton, G., et al. (2015). Angioplasty for Renovascular Hypertension in 78 Children. Arch. Dis. Child. 100, 474-478. doi:10.1136/archdischild-2013-305886

Kaths, J. M., Echeverri, J., Linares, I., Cen, J. Y., Ganesh, S., Hamar, M., et al. (2017). Normothermic Ex Vivo Kidney Perfusion Following Static Cold Storage-Brief, Intermediate, or Prolonged Perfusion for Optimal Renal Graft Reconditioning? Am. J. Transpl. 17, 2580-2590. doi:10.1111/ajt.14294

Kim, M. J., Lee, K. W., Park, J. B., and Kim, S. J. (2017). Hand-Assisted Laparoscopic Nephrectomy and Auto-Transplantation for a Hilar Renal Artery Aneurysm: A Case Report. VSI 33, 84-87. doi:10.5758/vsi.2017.33.2.84

Kishore, T. A., Kuriakose, M. J., Pathrose, G., Raveendran, V., Kumar, K. V., and Unni, V. N. (2020). Robotic Assisted Kidney Transplantation in Grafts with Multiple Vessels: Single center Experience. Int. Urol. Nephrol. 52, 247-252. doi:10.1007/s11255-019-02305-z

Kubota, R., Araki, M., Wada, K., Kawamura, K., Maruyama, Y., Mitsui, Y., et al. (2020). Robotic Renal Autotransplantation: A Feasibility Study in a Porcine Model. Acta Med. Okayama 74, 53-58. doi:10.18926/AMO/57953

Kumar, A., Chaturvedi, S., Gulia, A., Maheshwari, R., Dassi, V., and Desai, P. (2018). Laparoscopic Live Donor Nephrectomy: Comparison of Outcomes Right versus Left. Transplant. Proc. 50, 2327-2332. doi:10.1016/ j.transproceed.2018.03.034 
Lin, D., Xiang, T., Qiu, Q., Leung, J., Xu, J., Zhou, W., et al. (2020). Aldehyde Dehydrogenase 2 Regulates Autophagy via the Akt-mTOR Pathway to Mitigate Renal Ischemia-Reperfusion Injury in Hypothermic Machine Perfusion. Life Sci. 253, 117705. doi:10.1016/j.lfs.2020.117705

Madhavan Nampoothiri, K., Nair, N. R., and John, R. P. (2010). An Overview of the Recent Developments in Polylactide (PLA) Research. Bioresour. Techn. 101, 8493-8501. doi:10.1016/j.biortech.2010.05.092

Meier, R. P. H., Piller, V., Hagen, M. E., Joliat, C., Buchs, J.-B., Nastasi, A., et al. (2018). Intra-Abdominal Cooling System Limits Ischemia-Reperfusion Injury during Robot-Assisted Renal Transplantation. Am. J. Transpl. 18, 53-62. doi:10.1111/ajt.14399

Menon, M., Sood, A., Bhandari, M., Kher, V., Ghosh, P., Abaza, R., et al. (2014). Robotic Kidney Transplantation with Regional Hypothermia: A Step-by-step Description of the Vattikuti Urology Institute-Medanta Technique (IDEAL Phase 2a). Eur. Urol. 65, 991-1000. doi:10.1016/j.eururo.2013.12.006

Ölçücüoğlu, E. (2020). Comparing the Complications of Laparoscopically Performed Simple, Radical and Donor Nephrectomy. Turk J. Med. Sci. 50, 922-929. doi:10.3906/sag-1910-120

Perkins, S. Q., Giffen, Z. C., Buck, B. J., Ortiz, J., Sindhwani, P., and Ekwenna, O. (2018). Initial Experience with the Use of a Robotic Stapler for RobotAssisted Donor Nephrectomy. J. Endourol. 32, 1054-1057. doi:10.1089/ end.2018.0461

Pomy, B., Glousman, B., and Macsata, R. (2020). Management of Bilateral Renal Artery Aneurysms with Laparoscopic Nephrectomy, Ex Vivo Reconstruction, and Autotransplantation in a Woman Planning Pregnancy. J. Vasc. Surg. Cases, Innov. Tech. 6, 126-128. doi:10.1016/j.jvscit.2020.01.006

Raborn, J., McCafferty, B. J., Gunn, A. J., Moawad, S., Mahmoud, K., Aal, A. K. A., et al. (2020). Endovascular Management of Neurofibromatosis Type I-Associated Vasculopathy: A Case Series and Brief Review of the Literature. Vasc. Endovascular Surg. 54, 182-190. doi:10.1177/1538574419885257

Ruiz, M., Hevia, V., Fabuel, J.-J., Fernández, A.-A., Gómez, V., and Burgos, F.-J. (2017). Kidney Autotransplantation: Long-Term Outcomes and Complications. Experience in a Tertiary Hospital and Literature Review. Int. Urol. Nephrol. 49, 1929-1935. doi:10.1007/s11255-017-1680-1

Safdar, O., Alaifan, F., Alshammakh, S., Hakami, M., and Alghaithi, D. F. (2020). Diagnostically Challenging Case of Renal Artery Stenosis in a Pediatric Patient. Cureus 12, e6538. doi:10.7759/cureus.6538

Samuels, J. A., Zavala, A. S., Kinney, J. M., and Bell, C. S. (2019). Hypertension in Children and Adolescents. Adv. Chronic Kidney Dis. 26, 146-150. doi:10.1053/ j.ackd.2019.02.003

Seitz, C., and Hiess, M. (2016). Robot-assisted Renal Surgery: Current Status and Future Directions. Robot Surg. 3, 1-12. doi:10.2147/RSRR.S71328

Siena, G., Vignolini, G., Mari, A., Li Marzi, V., Caroassai, S., Giancane, S., et al. (2019). Full Robot-Assisted Living Donor Nephrectomy and Kidney Transplantation in a Twin Dedicated Operating Room: Initial Experience from a High-Volume Robotic Center. Surg. Innov. 26, 449-455. doi:10.1177/ 1553350619835429
Smith, M., Lazar, A., Morrissey, N., and Ratner, L. (2020). Laparoscopic Nephrectomy with Ex Vivo Repair of Aneurysm and Autotransplantation. J. Vasc. Surg. Cases, Innov. Tech. 6, 24-26. doi:10.1016/j.jvscit.2019.11.009

Spinoit, A.-F., Moreels, N., Raes, A., Prytula, A., De Groote, R., Ploumidis, A., et al. (2019). Single-setting Robot-Assisted Kidney Transplantation Consecutive to Single-Port Laparoscopic Nephrectomy in a Child and Robot-Assisted LivingRelated Donor Nephrectomy: Initial Ghent Experience. J. Pediatr. Urol. 15, 578-579. doi:10.1016/j.jpurol.2019.08.005

Territo, A., Piana, A., Fontana, M., Diana, P., Gallioli, A., Gaya, J. M., et al. (2021). Step-by-step Development of a Cold Ischemia Device for Open and RoboticAssisted Renal Transplantation. Eur. Urol. 21, 01795-1804. doi:10.1016/ j.eururo.2021.05.026

Tiong, H. Y., Goh, B. Y. S., Chiong, E., Tan, L. G. L., and Vathsala, A. (2018). Robotic Kidney Autotransplantation in a Porcine Model: a Procedure-specific Training Platform for the Simulation of Robotic Intracorporeal Vascular Anastomosis. J. Robotic Surg. 12, 693-698. doi:10.1007/s11701-018-0806-5

Tyler, B., Gullotti, D., Mangraviti, A., Utsuki, T., and Brem, H. (2016). Polylactic Acid (PLA) Controlled Delivery Carriers for Biomedical Applications. Adv. Drug Deliv. Rev. 107, 163-175. doi:10.1016/ j.addr.2016.06.018

Urbanellis, P., Hamar, M., Kaths, J. M., Kollmann, D., Linares, I., Mazilescu, L., et al. (2020). Normothermic Ex Vivo Kidney Perfusion Improves Early DCD Graft Function Compared with Hypothermic Machine Perfusion and Static Cold Storage. Transplantation 104, 947-955. doi:10.1097/ TP.0000000000003066

Xu, Y., Yuan, Y., Cai, Y., Li, X., Wan, S., and Xu, G. (2020). Use 3D Printing Technology to Enhance Stone Free Rate in Single Tract Percutaneous Nephrolithotomy for the Treatment of Staghorn Stones. Urolithiasis 48, 509-516. doi:10.1007/s00240-019-01164-8

Conflict of Interest: The authors declare that the research was conducted in the absence of any commercial or financial relationships that could be construed as a potential conflict of interest.

Publisher's Note: All claims expressed in this article are solely those of the authors and do not necessarily represent those of their affiliated organizations, or those of the publisher, the editors, and the reviewers. Any product that may be evaluated in this article, or claim that may be made by its manufacturer, is not guaranteed or endorsed by the publisher.

Copyright $\odot 2022 \mathrm{Cui}, \mathrm{Wu}, \mathrm{He}$, Wang, Jiao and Zhang. This is an open-access article distributed under the terms of the Creative Commons Attribution License (CC BY). The use, distribution or reproduction in other forums is permitted, provided the original author(s) and the copyright owner(s) are credited and that the original publication in this journal is cited, in accordance with accepted academic practice. No use, distribution or reproduction is permitted which does not comply with these terms. 